\title{
Numerical Simulations of High Redshift Star Formation in Dwarf Galaxies
}

\author{
Konstantinos Tassis \\ Department of Astronomy, University of Illinois at Urbana-Champaign, \\ 1002 W. Green Street, Urbana, IL, US-61801 \\ tassis@astro.uiuc.edu \\ Tom Abel \\ Penn State University, University Park, PA, US-16802 \\ Greg L. Bryan \\ University of Oxford, Astrophysics, Keble Road, Oxford OX1 3RH, UK \\ Michael L. Norman \\ University of California, San Diego, CA, US-92093 La Jolla
}

\begin{abstract}
We present first results from three-dimensional hydrodynamic simulations of the high redshift formation of dwarf galaxies. The simulations use an Eulerian adaptive mesh refinement technique to follow the non-equilibrium chemistry of hydrogen and helium with cosmological initial conditions drawn from a popular $\Lambda$-dominated cold dark matter model. We include the effects of reionization using a uniform radiation field, a phenomenological description of the effect of star formation and, in a separate simulation, the effects of stellar feedback. The results highlight the effects of stellar feedback and photoionization on the baryon content and star formation of galaxies with virial temperatures of approximately $10^{4} \mathrm{~K}$. Dwarf sized dark matter halos that assemble prior to reionization are able to form stars. Most halos of similar mass that assemble after reionization do not form stars by redshift of three. Dwarf galaxies that form stars show large variations in their gas content because of stellar feedback and photoionization effects. Baryon-to-dark matter mass ratios are found to lie below the cosmic mean as a result of stellar feedback. The supposed substructure problem of CDM is critically assessed on the basis of these results. The star formation histories modulated by radiative and stellar feedbacks are discussed. In addition, metallicities of individual objects are shown to be naturally correlated with their mass-to-light ratios as is also evident in the properties of local dwarf galaxies.
\end{abstract}


Subject headings: cosmology: theory - early universe - galaxies: formation

\section{Introduction}

Radiative and stellar feedback have long been recognized as an essential ingredient in galaxy formation and they are thought to play an important role in determining the properties of dwarf galaxies. The smallest dark matter halos are thought to be the sites for the formation of the first stars (Couchman and Rees 1986; Tegmark et al. 1997, Haiman and Loeb 1997; Abel et al. 1998; Bromm, Coppi and Larson 1999; Abel, Bryan and Norman $2000,2001)$. Whether these smallest objects will allow a significant fraction of the baryons to participate in primordial star formation is unclear because of the effect of complex radiative feedback processes on molecular hydrogen formation (Haiman, Abel and Rees 2000; Ciardi, Ferrara, Abel 2000; Mahacek, Bryan and Abel 2001, Glover and Brand 2001). However, the early formation of even a few stars in such low-mass objects may significantly affect the evolution of their baryon content due to supernova explosions driving a significant fraction of the gas away from the gravitational potential of the host.

Stellar feedback was first discussed in the context of primordial globular cluster formation by Peebles and Dicke (1968). In the context of hierarchical structure formation, the possibility that supernovae may remove a significant fraction of the gas present in dwarf galaxies was discussed by Dekel \& Silk (1986) and by Couchman \& Rees (1986). Further analytical treatment of such supernova-driven winds was presented by Babul \& Rees (1992) and Efstathiou (1992).

Star formation and feedback have also been studied in the context of cosmological structure formation simulations. However, the efficiency of supernova energy feedback in driving the gas out of the dark matter potentials was found to depend on the details of the feedback prescription: Katz (1992) and Weinberg, Hernquist \& Katz (1997) found that the properties of their simulated galaxies were insensitive to the inclusion of star formation and feedback to the simulations. Navarro \& White (1993) and Navarro \& Steinmetz (1999, 2000)

found that the effect of supernova feedback depends on whether the energy returned to the ISM is in the form of thermal or kinetic energy. In the former case, the energy was quickly radiated away and the effect of feedback was minimal, while in the latter case feedback was efficient in driving gas away from the dark matter potentials and in suppressing star formation. In addition, Mac Low \& Ferrara (1999) and Navarro \& Steinmetz (2000) found that the effect of feedback depends on the total mass of the system under consideration, with the low-mass systems being affected much more than the higher-mass systems. 
Energy feedback from supernovae is also believed to have a significant effect on the metallicities of dwarf galaxies. The idea of metals being driven away from galaxies is supported by observations of the chemical enrichment of the IGM (e.g. Ellison et al. 2001 and references therein) as well as observations of dwarf galaxies in he Local Group. Prada \& Burkert (2002) have summarized a correlation between mass-to-light ratio and metallicity of dwarf spheroidals, which they attribute to metal loss being more efficient for objects with shallower potential wells.

The ability of supernova-driven winds to remove metals from galaxies has been studied by Mac Low \& Ferrara (1999) and by Aguirre et al. (2001). Mac Low \& Ferrara (1999) modeled the effects of repeated supernova explosions from starbursts in dwarf galaxies with hydrodynamic simulations performed in a pre-determined dark matter gravitational potential and with an initial baryon-to-gas mass ratio extrapolated from observations of higher mass systems. Their results suggest that galactic winds are very efficient in removing metals from dwarf galaxies, with the effect being more pronounced for the least massive objects. For larger energy ejection rates (Mac Low \& Ferrera considered star formation rates only up to about $0.004 M_{\odot} /$ year), the gas in the galaxy can be completely blown away (Mori, Ferrara \& Madau 2002).

Aguirre et al. (2001) used results from completed N-body + hydrodynamic simulations including star formation which, however, did not directly incorporate the result of feedback, and assumed a series of ad hoc algorithms to reconstruct the metal enrichment process starting from the star formation history in each simulated object. They found that most galaxies at redshift $\sim 3$ should exhibit winds driving metals away from their gravitational potentials and enriching the IGM with metals. Note that such winds can now be studied directly using aborption spectra of quasars behind Lyman break galaxies (Adelberger et al. 2002).

An additional factor affecting the baryon contents of dwarf galaxies is photoionization. The Jeans mass prior to reionization is approximately a factor $10^{5}$ smaller than after, i.e. the minimum masses of dark matter halos that can confine baryons are much less at times before the intergalactic medium has been ionized.

Efstathiou (1992) and Babul \& Rees (1992) discussed analytically the possibility that a photoionizing background might significantly affect the formation of dwarf galaxies and may be the mechanism preventing the collapse of most of the baryonic material into subgalactic objects at high redshifts. Thoul and Weinberg (1996) showed, using a one-dimensional hydrodynamics code, that photo-ionization affects the collapse of gas into dark halos with velocity dispersions even in excess of $30 \mathrm{~km} \mathrm{~s}^{-1}$. 
Kepner, Babul and Spergel (1997) have looked at the formation of molecular hydrogen in the cores of proto-dwarf galaxies and Kepner et al. (1999) investigated observational consequences of the picture of delayed dwarf galaxy formation. The scenario for the formation of dwarf galaxies outlined by Ferrara and Tolstoy (2000) also takes into account the feedback from photo-ionization. In their model the first stars in observed dwarfs form prior to reionization. Star formation at later epochs originates from new gas infall as the meta-galactic UV background radiation field decreases. Gnedin (2000) studied the effect of photoionization on the gas fraction of low-mass objects and found a deviation from the universal baryon fraction at low masses.

Many of these papers, as well as Ciardi et al. (2000), did implicitly predict that not all the substructure seen in dark matter-only simulations should also host luminous objects. Images of multiply lensed quasars may be used to constrain the amount of dark substructure in galaxies (Metcalf and Madau 2001). Interestingly, existing data on such systems favors the existence of such dark matter substructure (Metcalf and Zhao 2002, Dalal and Kochanek 2002).

In this paper, we use high-mass resolution, 3-dimensional hydrodynamic AMR simulations to examine the effect of stellar feedback and photoionization on the gas content, star formation histories and metallicities of dwarf galaxies down to a redshift of 3. Star formation and feedback are included using a phenomenological recipe. Since star formation, metal and energy feedback are followed concurrently with the dark matter and gas evolution, our simulations do not require invoking any assumptions on the nature of supernova driven winds (as in Aguirre et al. (2001)) or on the shape of the dark matter gravitational potentials (as in Mac Low \& Ferrara (1999)). In addition, grid based methods are known to be superior to particle techniques when a multi-phase medium needs to be resolved (Croft et al. 2001, Pearce et al. 2000, Ritchie \& Thomas 2001, and references therein). Here, we will only discuss aspects of the simulation that are expected to be insensitive to the details of the star formation algorithm.

Our paper is organized as follows: In $\S 2$ we describe the simulations carried out as well as relevant aspects of our implementation of star formation and feedback. In $\S 3$ we discuss the global star formation history in our cube before and after reionization. In $\S 4$ we present the effects of feedback and reionization on the baryon contents, star formation histories and metallicities of individual halos. Finally, we discuss our findings in $\S 5$. 


\section{Simulations}

We have used the 3-D AMR cosmology code described by Bryan \& Norman (1997a), Norman and Bryan (1999), Bryan, Abel and Norman (2001). The AMR algorithm used is similar to the one described by Berger and Collela (1989). It utilizes an adaptive hierarchy of grid patches at various levels of resolution. Each rectangular grid patch covers the region of space in its parent grid needing higher resolution, and may itself become the parent grid to an even higher resolution child grid. Initial and boundary conditions are obtained by interpolation from the parent grid when the child grid is created.

In the simulations presented in this paper, we limited the refinement to 9 levels in a $128^{3}$ top grid. However, at the end of each run, only 7 levels of refinement had been used, so our simulations were not resource bounded. We have used a box size of $10 h^{-1} \mathrm{Mpc}$ comoving, which was chosen as a compromise between high mass resolution and reasonable volume. The maximum spatial resolution (i.e. minimum cell size) achieved was $610 h^{-1}$ comoving pc or better than 150 proper pc for the redshifts simulated. The dark matter mass resolution was $M_{\mathrm{DM}} \approx 5 \times 10^{7} M_{\odot}$.

While this resolution is insufficient to model the detailed internal structure of the galaxies and the way that feedback works on pc scales, it is enough to correctly model the conversion of SN energy into high-velocity outflows which affect the gas surrounding (and infalling into) galaxies. For a more detailed examination of the impact of a burst of SN within a single high-redshift halo of this mass, see Mori, Ferrara \& Madau (2002); their results support our findings that massive outflows are common around starbursting galaxies.

The simulations were set up as a flat $\Lambda$ CDM model with $\Omega_{\Lambda}=0.7$ and $\Omega_{m}=0.3$. The total matter density consists of a baryonic and a dark matter component, where $\Omega_{b}=0.04$ and $\Omega_{D M}=0.26$. The present-day value of the dimensionless Hubble parameter was $h=0.67$ in units of $100 \mathrm{~km} / \mathrm{s} / \mathrm{Mpc}$. The simulations were initialized at redshift 60 and terminated at redshift 3. The cosmological density field was initialized using power spectra for the dark matter and the baryons given by the fitting functions of Eisenstein \& $\mathrm{Hu}$ (1999).

Our simulations have allowed for star formation, however radiation feedback from the formed "stars" was not taken into account - instead, the simulation implements a homogeneous radiation field of Haardt \& Madau type (Haardt and Madau 1996), with an index of -1.5 , i.e. full reionization occurs in our models at $z \sim 6 .{ }^{1}$ We also solve the non-equilibrium chemistry and cooling from hydrogen and helium and their ions (Abel et al. 1997, Anninos

\footnotetext{
${ }^{1} \mathrm{~A}$ more refined study of cosmological reionization using the galaxies formed in our no-feedback run has been presented in Razoumov et al (2002).
} 
et al. 1997). Cooling from metal lines has been approximated by assuming collisional equlibrium. However, it should be noted that in the case of galaxy formation radiative processes may strongly affect the ionization balances and hence the cooling rates.

In order to allow for star formation, given that it is not feasible to directly simulate the formation of individual stars since the range of scales and additional physics required to do so is prohibitive, a parametric method was adopted which attempts to model star-forming regions following a prescription similar to the one introduced by Cen and Ostriker (1992). A detailed description of the implementation of the star-formation and feedback scheme can be found in O'Shea et al. (2002).

Groups of stars forming are treated as pressureless particles which return feedback to the surrounding medium (star particles). For a star particle to be created, the following criteria need to be met:

1. The baryon density at the particular grid where the star particle is to be created exceeds the mean density in the universe by a predefined factor, set to be equal to 100 in our simulation.

2. The gas in the particular grid cell has $\nabla \cdot \mathbf{v}<0$, i.e. the gas is infalling rather than expanding (or $T<10^{4} \mathrm{~K}$ ).

3. The cooling timescale for the gas is smaller than the dynamical collapse timescale.

4. The baryonic mass in the cell exceeds the local Jeans mass.

The mass of the star particle to be created is calculated as

$$
m_{*}=f_{* \mathrm{eff}} m_{b} \frac{\Delta t}{t_{\mathrm{dyn}}}
$$

where $m_{b}$ is the baryon mass of the cell, $\Delta t$ is the duration of a timestep, $t_{\text {dyn }}$ is the dynamical collapse timescale and $f_{* \text { eff }}$ is the "star formation efficiency" (fraction of mass of the initial cloud which is converted into stars), set equal to $80 \%$ in this simulation. This value of $f_{* \text { eff }}$ is significantly larger than values expected in realistic systems but we have used it in order to investigate the limiting case as to the maximal effect of feedback. Additionally, we never allow more than $90 \%$ of the mass in a cell to be converted to stars.

Conditions 1-4 are necessary but not sufficient for a star particle to be formed: in order to prevent the formation of an excessive number of star particles which would require a prohibitively high amount of computational resources in order to be followed, a minimum star particle mass $m_{* \min }$ is introduced. A star particle is formed in a cell fulfilling the criteria 
1-4 only if $m_{*}$ exceeds this minimum mass. The value of $m_{* \min }$ we used was $10^{6} \mathrm{M}_{\odot}$. However, since the minimum mass requirement is associated with computational resources limitations rather than underlying physics, a "bypass mechanism" for this restriction was included in the algorithm to account for small-mass star particles which should have been created on physical grounds: there is a certain probability per time step that a star particle with mass lower than the minimum mass threshold is created in a cell fulfilling the requirements 1-4 but not the minimum mass requirement. This probability is equal to the ratio of the mass of the star particle that would be created in the cell over the minimum star particle threshold.

To avoid the unphysical situation of forming a star particle (corresponding to a cluster of stars) instantaneously, a more reasonable star formation history has been assigned to each cold collapsing cloud. Therefore, each star particle $i$ is assumed to have a time-dependent star formation rate of

$$
\psi_{i}(t)=m_{*} \frac{t-t_{\mathrm{cr}}}{\tau^{2}} \exp \left(-\frac{t-t_{\mathrm{cr}}}{\tau}\right)
$$

where $t_{\text {cr }}$ is the time of creation of the star particle and $\tau=\max \left[t_{\mathrm{dyn}}, 10 \mathrm{Myrs}\right]$, with $t_{\mathrm{dyn}}$ being the dynamical time from equation (1). Thus, one observes a rapid star formation after a dynamical time with an exponential tail at longer times. If $t_{\mathrm{dyn}}$ is shorter than the small but non-zero life-span of massive stars, an estimate of the latter (here taken to be 10 Myrs) is used in place of $t_{\mathrm{dyn}}$.

The star particles formed give energy, metals and momentum feedback to the baryon gas. The energy equivalent to $10^{-5}$ of the rest mass energy of the stars generated is converted into thermal energy (this corresponds to $1 \mathrm{SNe}$ of $10^{51}$ erg for every $55 \mathrm{M}_{\odot}$ of stars being formed) over a time period corresponding to the dynamical time from equation (1) and is put directly into the thermal energy of the cell in which the star particle resides. Metals are also returned to the gas over the same time period and the yield (mass of metals generated per mass of stars created) is 0.02 . The mass fraction of created stars which is returned to the gas phase is $25 \%$. The values for the parameters of our star formation algorithm are taken from Cen \& Ostriker (1992).

In order to better understand which effects in the output of our simulation can be attributed to the feedback from the star particles to the baryonic gas, we have run a second, control simulation, with the exact same cosmological parameters and initial conditions as our original simulation, in which stellar particles are born following the exact same recipe as before, but they do not return energy, momentum or metals to the gas. In the following sections we will present and compare results from both simulations. We will refer to the original simulation (the one which allows for stellar feedback) as "feedback run" and to the control simulation (the one without stellar feedback) as "no-feedback run". It is interesting to note that the two simulations presented here are both limiting cases as far as feedback is 
concerned, in the sense that in our original simulation the feedback from each individual star particle is rather overestimated compared to theoretical expectations for realistic physical systems, while in our second (control) simulation the feedback is entirely absent.

\section{The Global Star Formation History}

Fig. 1 gives the global star formation rate in our simulated box (in $\mathrm{M}_{\odot}$ per year per comoving $\mathrm{Mpc}^{3}$ ) as a function of redshift. In the same plot, we have overplotted observational data points compiled by Blain (2001). The observational points are meant to give a feeling of the magnitude of the star formation rate density in our box in terms of the corresponding quantity observed in the real universe rather than serve as an observational test for our model. The unrealistically large value of the star formation efficiency, combined with the small box size, can result to rapid gas depletion, causing the cosmic star formation rate to peak at too high a redshift and therefore a direct comparison between our results and the observational data points can be misleading. With these caveats in mind, it is nevertheless interesting to note that the magnitude of the cosmic star formation rate as derived from our simulations is comparable with that of the observational data over a considerable range of redshifts.

The overall star formation rate is significantly larger in the no-feedback case: allowing SN to inject energy into the ISM causes strong galactic winds which drive the baryonic gas away from the halos, while at the same time contributing to the increase of the temperature and pressure in the ISM, hindering the process of star formation. As a result, the global star formation rate can take much larger values in the no-feedback run.

In both runs, there is a change of slope of the cosmic star formation rate at around the epoch of re-ionization, an effect associated with both reionization and gas depletion. The radiation field is implemented in the same way in both runs and is independent of stellar feedback, so reionization is felt equally in both simulations. However, the decrease of the star formation activity at low redshifts is much more prominent in the feedback run, due to the extra effect of gas depletion of halos. 


\section{Dwarf Galaxies}

\subsection{The Inventory of simulated Dwarf Galaxies}

We find and define the centers of halos using HOP (Eisenstein \& Hut 1998) on the dark matter. A halo is then defined as the spherical region around these centers which has an average dark matter density $\Delta_{\text {vir }}(z)$ times the critical density $\rho_{\text {crit }}(z)$ (Bryan and Norman 1998). We require a minimum of 100 dark matter particles (corresponding to a dark matter mass of $\sim 10^{9} M_{\odot}$ ) in order to identify an overdense region as a dark matter halo. Subhalos (objects identified as halos by our algorithm but which are included in larger virialized structures) have been removed from the resulting set of halos.

Fig. 2 shows the variations of the baryonic content (i.e. stars + gas) of the simulated galaxies at redshift $z=3$ as well as their gas and stellar contents vs total mass, for both the feedback run (left panel) and the no-feedback run (right panel). Open circles represent halos with no stellar content while crosses represent halos with stellar content. The dashed line corresponds to the ratio $\Omega_{b} / \Omega_{m}$ (average ratio of baryon density over total matter density in the universe).

In the no-feedback run, the total baryon content is closely scattered around the $\Omega_{b} / \Omega_{m}$ line, as seen in previous $\Lambda$-CDM simulations (e.g. Pearce et al. 2001). The deviation from the cosmic mean at low masses is due to the effect of photoionization on the baryon contents of dark matter halos and follows the shape also found by Gnedin (2000). On the contrary, in the feedback run, even the largest halos at $\mathrm{z}=3$ consistently contain only a small fraction of the baryons corresponding to $\Omega_{b} / \Omega_{m}$, which is also suggested by the properties of observed dwarf galaxies in the local group (e.g. Mateo 1998 and references therein) This significant difference between the results of the two runs indicates that low galaxy baryon contents can occur as a result of energy and momentum feedback from supernovae to the baryon gas: the latter is driven out of the potential well of the dark matter halos, leaving behind galaxies of lowered baryon content.

We can draw additional support for this picture by comparing the two panels in the middle row of figure 2 which displays the ratio of gas mass over total mass for the $\mathrm{z}=3$ halos. In the left panel figure, which corresponds to the case with stellar feedback, the scatter of halos towards low gas mass values is much larger than in the no-feedback case. This fact, considered in the framework of the scenario described above, is no surprise: in the no-feedback run the only mechanisms for gas depletion in a halo are the pressure from photoionization (reionization), tidal stripping and the conversion of baryon gas to stars. In the simulation where feedback is taken into account, gas can be lost both to star formation and to the intergalactic medium because of galactic winds driven by supernovae. The additional 
gas depletion mechanism drives galaxies towards lower gas content values in the feedback run.

By comparing the two bottom panels of figure 2 we can draw conclusions concerning the stellar population of halos in the feedback and no-feedback runs. A first obvious result is the existence of a large scatter in the total stellar content of galaxies both in the nofeedback and the feedback case. However, if we compare the galaxies with the largest stellar contents in both simulations, we find that the no-feedback run galaxies have much larger stellar contents, with the ratio of stellar mass over total mass reaching the $\Omega_{b} / \Omega_{m}$ value. This result is consistent with the much higher value of overall star formation rate in the nofeedback run when compared to that of the feedback run. Thus stellar feedback is a factor that clearly hinders star formation through the depletion of baryonic gas and the increase of the temperature and pressure of the ISM.

It is also interesting to note that dark halos which do not host stars (open circles in Fig. 2) all have relatively low masses. This can be understood within a hierarchical clustering scenario in terms of the inability of such small halos to hold photo-ionized gas.

An additional effect of feedback is that by $z=3$ there already exists a population of halos with no stellar content whose baryon gas content is also extremely low (as low as $10^{-6} \times$ $\left.M_{\text {dark matter }}\right)$. The observational detection of such halos which are practically "emptied" from baryonic gas would be extremely difficult. This result could at least partially explain the deficit of low-mass satellite galaxies in observations in the Local Group as compared to the expectations from CDM cosmological simulations (Moore et al. 1999, Klypin et al. 1999). The difference in mass to light ratios at small masses caused by reionization has also been investigated by Somerville (2002) and Benson et al. (2002). Integration of the effect of stellar feedback to such simulations naturally drives a portion of the low-mass systems to baryon contents low enough to render them unobservable by means other than their dynamical effects.

The morphology and complexity of the gas outflows responsible for the modification of the baryon contents of our simulated halos are demonstrated in figures 3 and 4 . In Fig. 3 the projection along the $\mathrm{x}$-axis of the gas density in the simulated cube is plotted in a logarithmic scale for redshifts 8, 5 and 3, and for both the feedback and the non-feedback runs. The color scale for $\log \left[\rho_{\text {gas }} /\left(M_{\odot} /\right.\right.$ proper $\left.\left.\mathrm{Mpc}^{2}\right)\right]$ ranges from 11.1 to 13.6 for all snapshots. The deviation between the feedback and non-feedback behaviour is evident from $z=5$ and is most striking at $z=3$. Winds driven by the stellar feedback increase the temperature of the baryonic gas and drive the gas away from the galaxies in a rather asymmetrical fashion.

Fig. 4 shows the projection along the x-axis of the x-ray weighted temperature in our 
cube, again for redshifts 8, 5 and 3 and for both the feedback and non-feedback run. The color scale is again logarithmic, and extends from $10^{2}$ to about $10^{8} \mathrm{~K}$, and is the same for all snapshots. Feedback is shown to result in the creation of non-spherical bubbles of hot gas, which, by redshift 3, have a typical size of about 1 comoving Mpc. The bubbles overlap, resulting in a global increase of the mean temperature of the gas in the cube, from $\sim 2.2 \times 10^{4}$ $\mathrm{K}$ in the non-feedback case, to $\sim 5.6 \times 10^{4} \mathrm{~K}$ in the feedback run, for $\mathrm{z}=3$. The wind-blown bubbles also do noticeably sweep up material in shells ahead of them.

\subsection{Star Formation Histories of Dwarf Galaxies at $z \geq 3$}

\subsubsection{Galaxies with and without Stellar Content}

The dwarf galaxy stellar content is affected by both feedback and photoionization. To demonstrate the relative importance of the two effects, we plot, in Fig. 5, the fraction of halos with stellar content (i.e. the number of halos which do host stars over the total number of halos in this mass range) in three different mass ranges ( between $10^{9} \mathrm{M}_{\odot}$ and $3 \times 10^{9} \mathrm{M}_{\odot}$ for panel a, between $3 \times 10^{9} \mathrm{M}_{\odot}$ and $6 \times 10^{9} \mathrm{M}_{\odot}$ for panel b and between $6 \times 10^{9} \mathrm{M}_{\odot}$ and $2 \times 10^{10} \mathrm{M}_{\odot}$ for panel c) as a function of redshift. The results of the feedback run are represented by the dashed curves, while the results of the no-feedback run correspond to the solid curves.

Most low-mass halos (panel a) have not yet formed stars by $z=8$ but, as time progresses, the baryon density and Jeans mass criteria of our star formation algorithm are satisfied in an increasing number of these halos and therefore the fraction of halos with stellar content initially increases. However, between redshifts 5 and 6 , when photoionization occurs, the process is reversed. The temperature (and therefore the Jeans mass) increase, cooling rates and typical densities decrease, and star formation is inhibited. Halos which accumulate enough mass to enter this mass range after reionization do not form stars, and the fraction of halos with stars decreases (no-feedback run, solid curve). When feedback is added to this picture, it enhances the effect of reionization, by providing a second mechanism for increasing the temperature of the interstellar medium and decreasing the maximum densities reached in a given halo. The curve maximum occurs at a higher redshift and the fraction of halos with stars decreases even more at low z (feedback run, dashed curve).

In halos of higher virial masses and temperatures, the central baryon densities are less affected by photo-ionization from reionization (panels b and c). However, in the same mass ranges, feedback is effective in increasing the ISM temperature and inhibiting star formation and is responsible for the small decrease in the number of higher-mass halos hosting stars at low redshifts, which is present only in the feedback run (dashed curves). 


\subsubsection{Star Formation Rates of Individual Galaxies}

In Fig. 1 we have seen the effect of feedback on the global SFR, which is to hinder the SF process. We will now examine how the CSFR is distributed among halos of different SF rates. By extracting information on the characteristic SFR of the typical SF sites, we can infer whether the bulk of the SF occurs in sites of large SF activity which could be observable at high redshifts, or rather in many low-SFR galaxies which would be unobservable. If the latter is the case, then observations would tend to underestimate the CSFR at high $(>3)$ redshifts, as has been suggested by Lanzetta et al. (2002).

Fig. 6 shows the distribution of the number of galaxies per logarithmic star formation interval for both feedback and non-feedback simulations and for redshifts $\mathrm{z}=3,4,6$ and 8 . Star formation rates are measured in $\mathrm{M}_{\odot} \mathrm{yr}^{-1}$. In the case with stellar feedback (top panel of Fig. 6), as we move to lower redshifts, the peak of the distribution moves towards lower star formation values, a result of the depletion of halos of baryon gas as the latter is being driven away from the dark matter halo potential wells by stellar explosions. This effect is absent in the case of the no-feedback run (lower panel of Fig. 6). Thus, in the presence of stellar feedback, halos tend to shift towards lower SFR with advancing cosmic time. By $z=3$ the distribution of halos in our simulation per SFR interval peaks around $10^{-3} \mathrm{M}_{\odot} \mathrm{y}^{-1}$.

Although Fig. 6 clearly demonstrates that there are many more halos with low SFR by $\mathrm{z}=3$, this does not necessarily suggest that most $\mathrm{SF}$ activity takes place at low SFR halos. We would like to determine whether the low SFR halo population has a significant cumulative contribution to the global SF activity or if the latter is dominated by the few high-SFR sites. For this purpose, in Fig. 7 we plot the distribution of star formation activity per logarithmic star formation rate interval, $\mathrm{SFR} \times(\mathrm{dN} / \mathrm{d} \log \mathrm{SFR})$ vs $\log \mathrm{SFR}$. The area under this curve gives the total SFR at the specific redshift due to all star particles in our cube. Again the plots are for both the feedback and the non-feedback simulation and for redshifts $\mathrm{z}=3,4,6$ and 8. Star formation rates are measured in $\mathrm{M}_{\odot} \mathrm{yr}^{-1}$. In the no-feedback plot, the total SFR saturates after reionization (as also seen in Fig. 1), while in the feedback case there is a significant decline in the overall star formation rate as we progress to lower redshifts, due to the additional effect of gas depletion. It is also interesting that in the feedback run results, and at redshifts $<5$, a significant portion of the total star formation is indeed coming from low-SFR galaxies, potentially unobservable, which might lead to an underestimate of the cosmic star formation rate from observations of individual objects after reionization. However, before reionization, most SF activity comes from high-SFR halos and starburst-like events. In addition, when interpreting these results, the reader should keep in mind that due to the small volume of our simulation, the large galaxies with high SF are

under-represented: On the one hand, our small volume can only host 4 at most Lyman break 
galaxies. On the other hand, the high SF efficiency and the consequent rapid gas depletion force the SFR to peak at too high a redshift, so that by $z=3$ the SFRs of our simulated halos are being underestimated.

\subsection{Metallicities of Dwarf Galaxies at $\mathrm{z}=3$}

Prada \& Burkert (2002) have pointed out that there is a correlation between the massto-light ratio M/L of dwarf spheroidal Local Group satellites and their metallicities, $[\mathrm{Fe} / \mathrm{H}]$. They have found that

$$
\log (M / L)=-[F e / H]+\text { constant }
$$

In order to look for a similar correlation in the inventory of our simulated halos, we plot in Fig. 8 the logarithm of the ratio of total mass over stellar mass $\left(\log M / M_{\star}\right)$ vs the metallicity $[\mathrm{Fe} / \mathrm{H}]$ of each halo. To estimate the luminosities of our stellar particles, we have used $L \propto M_{\star}$. This is only an approximation, since $L$ depends on stellar age and metallicity as well; however, it is sufficient to estimate the trend of the $M / L$ ratio. Under the above assumption, $\log M / M_{\star}$ only differs from $\log M / L$ by an additive constant. In the same plot, we have drawn a line of slope -1 , as in the observational result of Prada \& Burkert (2002), with a shifted intercept (since we are plotting $M / M_{\star}$ rather than mass-tolight ratios). Although the scatter of the points is significant, one can observe the trend for the metallicity to decrease for increasing $M_{\text {total }} / M_{\star}$.

A large $M / L$ implies a small stellar population relative to the total mass and consequently less integrated metal enrichment of the ISM than systems with lower total mass to light ratios. In addition, small halos tend to blow out gas and metals and halt star formation before a high metallicity is reached within them. Thus, these systems tend to have lower metal abundances than more massive systems.

When comparing Fig. 8 with observations, one should keep in mind that the Local Group satellites are much more evolved objects, while the evolution of our simulated halos stops at $\mathrm{z}=3$. However, it is encouraging that the simulations do show this trend and the hope is that in the future it will be possible to simulate a larger volume at similar mass and spatial resolution down to redshift zero. 


\section{Discussion}

We have presented the first results of a large-scale three-dimensional cosmological hydrodynamic AMR simulation of a cube of comoving size $\sim 10 \mathrm{Mpc}$ living in a $\Lambda$ CDM universe with cosmological parameters $\left(\Omega_{\Lambda}, \Omega_{m}, h\right)=(0.7,0.3,0.67)$. Star formation was allowed for, according to a parametric prescription creating collisionless particle out of baryonic fluid condensations exceeding the local Jeans mass. We have compared the results of two runs, differing in the treatment of the stellar particles: in one case the latter did return energy, momentum and metal feedback to the interstellar medium, while in the other no stellar feedback was allowed for. The effects of reionization were studied by subjecting the baryon fluid to a homogeneous, Haardt \& Madau radiation field of index -1.5, independent of the cosmic star formation.

Over 300 galaxies are produced in our simulation over the mass range $3 \times 10^{9} \leq$ $M_{\text {tot }} / M_{\odot} \leq 7 \times 10^{11}$. The smallest dwarf galaxies in the local group show kinematic masses of $2 \times 10^{7} M_{\odot}$ (Mateo 1998 and references therein). However, these observed galaxies may be hosted in much more extended dark matter halos (Stoehr et al 2002). It seems premature to argue for a significant modification of the cold dark matter scenarios such as to ask for significant annihilation rates (Cen 2001) to accommodate for their existence. The star formation histories of the smallest dwarf galaxies are also essential in understanding the recently proposed substructure problem raised by Moore et al. (1999) and Klypin et al. (1999). It has been argued that the systems associated with the extensive dark matter substructure found in N-body simulations of galaxy formation would not be in accord with the observations. This was one of the reasons for the proposal of Spergel and Steinhardt (2000) that the dark matter may be self-interacting which has attracted significant theoretical interest (Burkert 2000; Kochanek and White 2000; Yoshida et al. 2000, and references therein). The connection between simulated dark matter substructure and observable quantities is a complex one and must depend dramatically on the intricacies of star formation and feedback in the small potential wells of interest (Bullock et al. 2000, Somerville 2002, Benson et al. 2002).

Our results have shown that feedback from stars is a factor which can deplete halos of baryonic gas and significantly reduce their baryon mass fraction. The gas depletion due to feedback results in halos having a baryon - to - dark matter mass ratio which is consistently below the cosmic mean, $\Omega_{b} / \Omega_{D M}$, even in the very high mass objects. In addition, feedback can result to the formation of dark matter halos empty of stars by $\mathrm{z}=3$, and a baryon fraction so low that their observation would be particularly difficult. The existence of such a population of dark dwarf halos could at least partially explain the problem of missing satellites in the Local Group.

We have found that feedback and reionization affect the star formation rate both globally 
and locally. The slope of the cosmic star formation history curve changes around the epoch of reionization with the effect being much more pronounced when feedback is present. In addition, most halos assembling after reionization do not form stars by $z=3$, an effect which is again enhanced by the presence of feedback. Furthermore, while before reionization most SF activity originates from high-SFR objects, after reionization there is a shift towards lower SFR halos as the source of most cosmic star formation. Thus, to the extent that this effect is not an artifact of rapid gas depletion due to our high SF efficiency, a significant amount of the total star formation at intermediate redshifts (3-5) might be taking place in low-SFR halos. This effect has been first discussed by Barkana and Loeb (2000) and is here reported in full cosmological hydrodynamical simulations.

Finally, we find that the metallicity of galaxies is correlated with the mass-to-light ratio, increasing for decreasing metallicity. This trend is consistent with observational results of dwarf spheroidals in the Local Group. However, most of the objects in our simulation will merge to make larger galaxies and only a very small fraction will survive as isolated dwarf sized galaxies until today. So the presented comparison again only serves as an illustration and has as yet little predictive quality for the formation of dwarf galaxies. Nevertheless, we find it very encouraging that these first results show that with yet larger simulations one will be able to make predictions and direct comparisons with properties of local dwarf galaxies.

K.T. would like to thank Brian O'Shea and Vasiliki Pavlidou for enlightening discussions. K.T. was partially supported by a scholarship from the Greek State Scholarships Foundation. T.A. is grateful for the hospitality of the Institute of Astronomy at Cambridge, UK where this work has been completed. We thank an anonymous referee for comments and suggestions which have improved this paper. Simulations were carried out on the Origin2000 system at the NCSA, University of Illinois at Urbana-Champaign with support from NSF grant AST-9803137 and NRAC allocation MCA98020N.

\section{REFERENCES}

Abel, T., Anninos, P., Zhang, Y., \& Norman, M. L. 1997, New Astronomy, 2, 181

Abel, T., Anninos, P., Norman, M. L., \& Zhang, Y. 1998, ApJ, 508, 518

Abel, T., Bryan, G. L. \& Norman, M. L. 2000, ApJ, 540, 39.

Abel, T., Bryan, G. L. \& Norman, M. L. 2001, Science, 10.1126/science.1063991.

Abel, T. \& Mo, H. J. 1998, ApJ, 494, L151 
Adelberger, K.L., Steidel, C.C., Shapley, A.E., Pettini, M. 2002, preprint astro-ph/0210314

Aguirre, A., Hernquist, L., Schaye, J., Weinberg, D. H., Katz, N., \& Gardner, J. 2001, ApJ, 560,599 .

Anninos, P., Zhang, Y., Abel, T., \& Norman, M. L. 1997, New Astronomy, 2, 209

Babul, A. \& Rees, M. J. 1992, MNRAS, 255, 346

Barkana, R. \& Loeb, A. 2000, ApJ, 539, 20

Benson, A. J., Frenk, C. S., Lacey, C. G., Baugh, C. M., \& Cole, S. 2002, MNRAS, 333, 177

Berger, M. J. \& Collela, P. 1989, J. Comp. Phys., 82, 64

Blain, A. W. 2001, Starburst Galaxies: Near and Far, 303 (also available at astro$\mathrm{ph} / 0011387)$

Bromm, V., Coppi, P. S., \& Larson, R. B. 1999, ApJ, 527, L5

Bryan, G. L. 1999, Computing in Science \& Engineering, March - April 1999, 46

Bryan, G. L., Abel, T., \& Norman, M. L. 2001, proceedings of Supercomputing 2001

Bryan, G. L. \& Norman, M. L. 1997a, in ASP Conf. Ser. 123, Computational Astrophysics, eds. D. A. Clarke \& M. J. West, (San Francisco: ASP), p. 363

Bryan, G. L. \& Norman, M. L. 1998, ApJ, 495, 80

Bryan, G. L. \& Norman, M. L. 1999, in Workshop on Structured Adaptive Mesh Refinement Grid Methods, IMA Volumes in Mathematics No. 117, ed. N. Chrisochoides, (New York: Springer-Verlag), p. 165

Bullock, J. S., Kravtsov, A. V., \& Weinberg, D. H. 2000, ApJ, 539, 517

Burles, S. \& Tytler, D. 1998, ApJ, 499, 699

Burkert, A. 2000, ApJ, 534, L143

Cen, R. \& Ostriker, J.P. 1992, ApJL, 399, 113

Cen, R. 2001, ApJ, 546, L77

Ciardi, B., Ferrara, A., \& Abel, T. 2000, ApJ, 533, 594

Ciardi, B., Ferrara, A., Governato, F., \& Jenkins, A. 2000, MNRAS, 314, 611 
Couchman, H. M. P. \& Rees, M. J. 1986, MNRAS, 221, 53

Eisenstein \& Hu 1999, ApJ, 511, 5

Ellison, S. L., Songaila, A., Schaye, J., \& Pettini, M. 2000, AJ, 120, 1175

Croft, R. A. C., Di Matteo, T., Davé, R., Hernquist, L., Katz, N., Fardal, M. A., \& Weinberg, D. H. 2001, ApJ, 557, 67

Dalal, N. \& Kochanek, C. S. 2002, ApJ, 572, 25

Dekel, A. \& Silk, J. 1986, ApJ, 303, 39

Efstathiou, G. 1992, MNRAS, 256, 43P.

Eisenstein, D. J. \& Hut, P. 1998, ApJ, 498, 137

Ferrara, A. \& Tolstoy, E. 2000, MNRAS, 313, 291

Glover, S. C. O. \& Brand, P. W. J. L. 2001, MNRAS, 321, 385

Gnedin, N.Y. 2000, ApJ, 542, 535

Haardt, F. \& Madau, P. 1996, ApJ, 461, 20

Haiman, Z., Abel, T., \& Rees, M. J. 2000, ApJ, 534, 11

Haiman, Z. \& Loeb, A. 1997, ApJ, 483, 21

Katz, N. 1992, ApJ, 391, 502

Kepner, J. V., Babul, A., \& Spergel, D. N. 1997, ApJ, 487, 61

Kepner, J., Tripp, T. M., Abel, T., \& Spergel, D. 1999, AJ, 117, 2063

Klypin, A., Kravtsov, A. V., Valenzuela, O., \& Prada, F. 1999, ApJ, 522, 82

Kochanek, C. S. \& White, M. 2000, ApJ, 543, 514

Lanzetta, K. M., Yahata, N., Pascarelle, S., Chen, H., \& Fernández-Soto, A. 2002, ApJ, 570, 492

Machacek, M. E., Bryan, G. L., \& Abel, T. 2001, ApJ, 548, 509

Mac Low, M.-M., \& Ferrara, A. 1999, ApJ, 513, 142

Madau, P., Pozzetti, L. \& Dickinson, M. 1998, ApJ, 498, 106 
Mateo, M. L. 1998, Ann. Rev. A \& A, 36, 435

Metcalf, R. B. \& Madau, P. 2001, ApJ, 563, 9

Metcalf, R. B. \& Zhao, H. 2002, ApJ, 567, L5

Mo, H. J., Mao, S., \& White, S. D. M. 1998, MNRAS, 295, 319

Moore, B.,Ghigna, S., Governato, F., Lake, G., Quinn, T., Stadel, J., \& Tozzi, P. 1999, ApJ, 524, L19

Mori, M., Ferrara, A. \& Madau, P. 2002, ApJ, 571, 40

Navarro, J. F. \& Steinmetz, M. 1997, ApJ, 478, 13.

Navarro, J.F \& Steinmetz, M. 2000, ApJ, 538, 477

Navarro, J.F \& White, S.D.M 1993, MNRAS, 265, 271

Norman, M. L. \& Bryan, G. L. 1999, ASSL Vol. 240: Numerical Astrophysics, 19

O’Shea, B., Bryan, G.L., Norman, M.L., Cen, R. \& Ostriker, J.P. 2002, to be submitted

Ostriker, J. P. \& Gnedin, N. Y. 1996, ApJ, 472, L3

Ostriker, J. P. \& Steinhardt, P. J. 1995, Nature, 377, 600

Pearce, F. R., Thomas, P. A., Couchman, H. M. P., \& Edge, A. C. 2000, MNRAS, 317, 1029

Pearce, F. R., Jenkins, A., Frenk, C. S., White, S. D. M., Thomas, P. A., Couchman, H. M. P., Peacock, J. A., \& Efstathiou, G. 2001, MNRAS, 326, 649

Peebles, P. J. E. \& Dicke, R. H. 1968, ApJ, 154, 891

Prada, F. \& Burkert, A. 2002, ApJ, 564, L73

Razoumov, A. O., Norman, M. L., Abel, T., \& Scott, D. 2002, ApJ, 572, 695

Ritchie, B. W. \& Thomas, P. A. 2001, MNRAS, 323, 743

Somerville, R. S. 2002, ApJ, 572, L23

Spergel, D. N. \& Steinhardt, P. J. 2000, PRL, 84, 3760

Steinmetz, M. \& Navarro, J. F. 1999, ApJ, 513, 555.

Stoehr, F., White, S. D. M., Tormen, G., \& Springel, V. 2002, MNRAS, 335, L84 
Tegmark, M., Silk, J., Rees, M. J., Blanchard, A., Abel, T., \& Palla, F. 1997, ApJ, 474, 1 Thoul, A. A. \& Weinberg, D. H. 1996, ApJ, 465, 608

Weinberg, D. H., Hernquist, L., \& Katz, N. 1997, ApJ, 477, 8.

Yoshida, N., Springel, V., White, S. D. M., \& Tormen, G. 2000, ApJ, 544, L87 


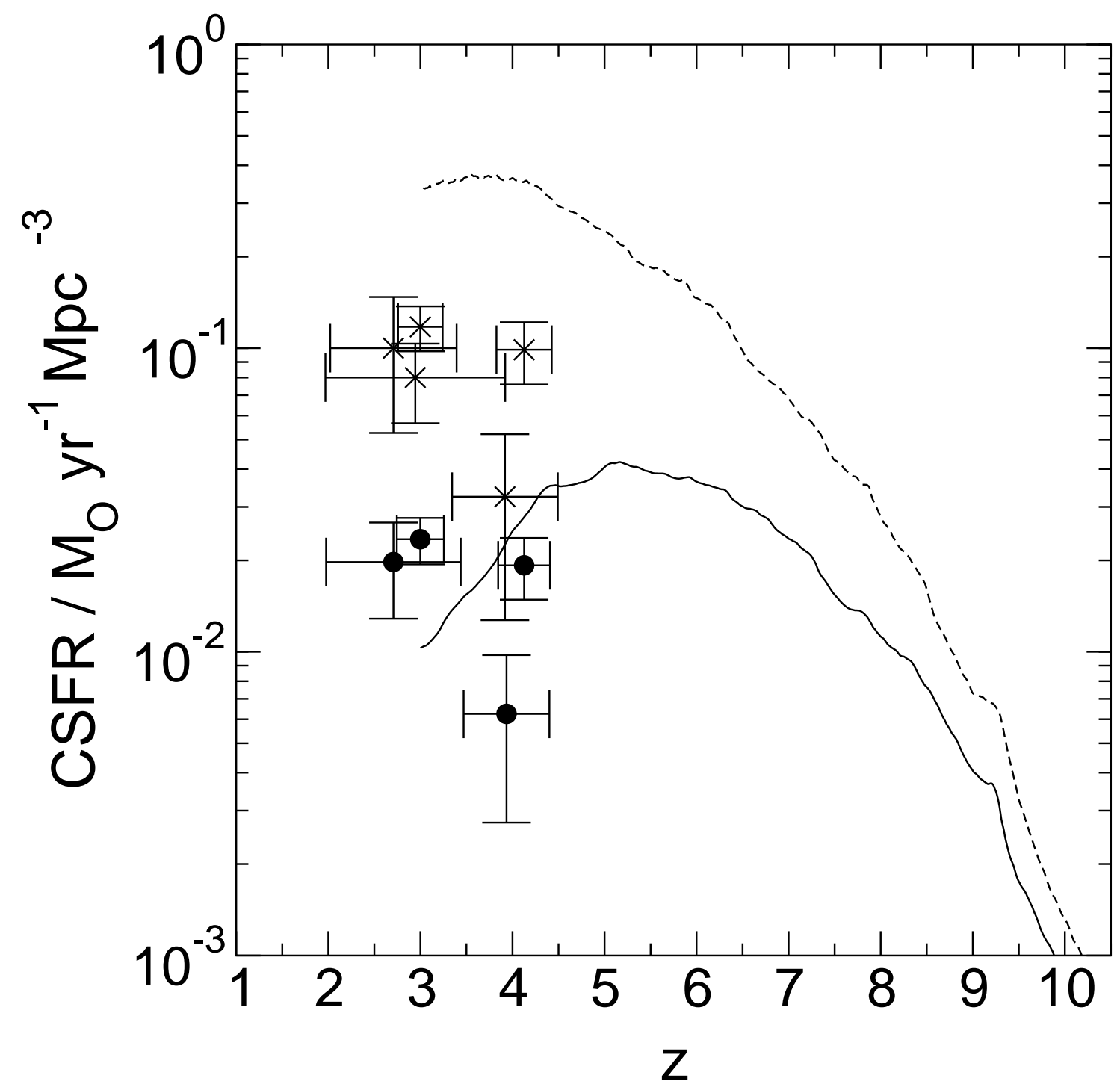

Fig. 1.- The global star formation history. The data points were taken from Blain (2001) and references therein. Filled circles represent measurements uncorrected for dust extinction, while diagonal crosses are all measurements which incorporate some correction for dust extinction. The solid line corresponds to the run with stellar feedback and the dashed line to the run without feedback. 


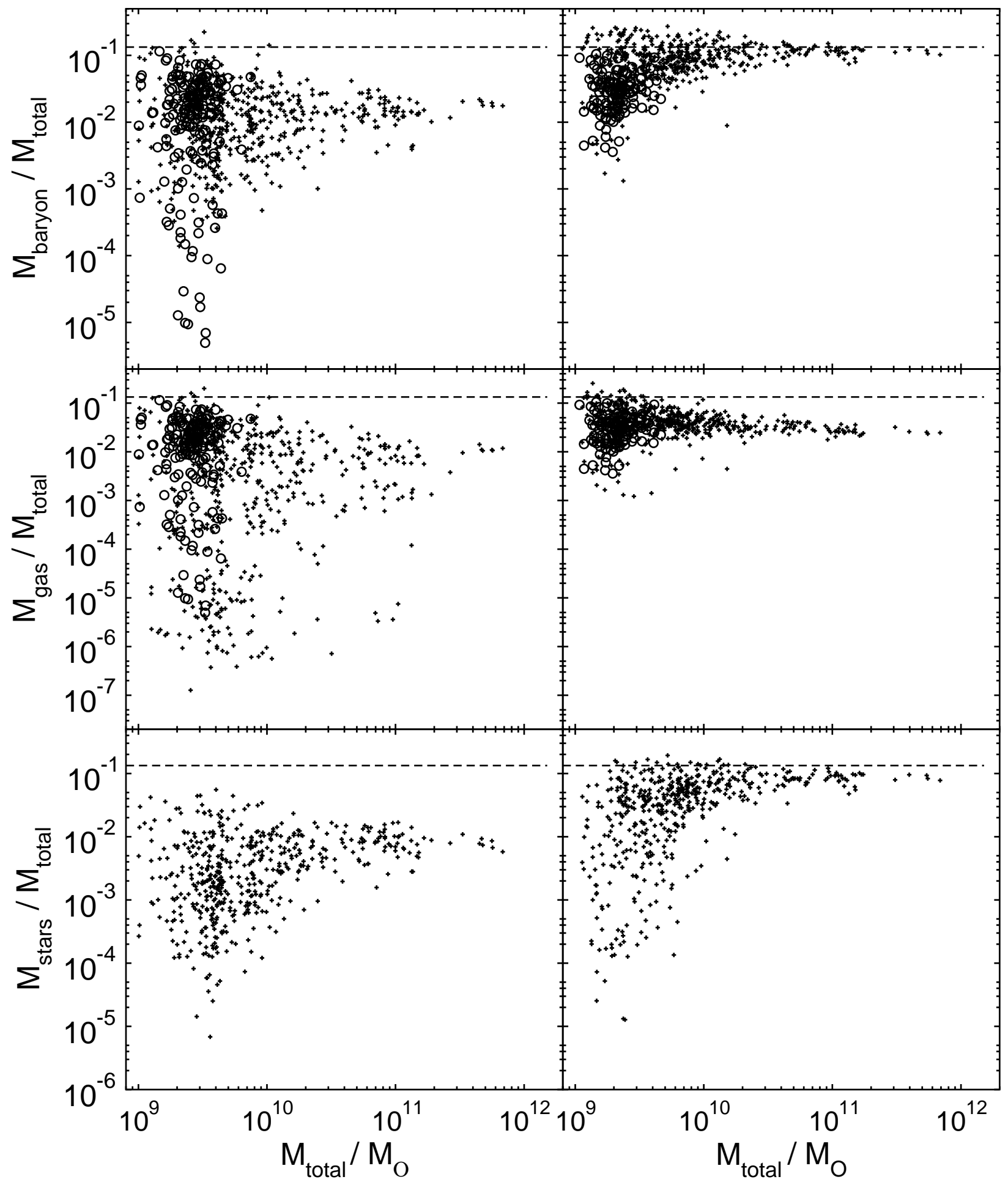

Fig. 2.- Baryonic content for all galaxies at redshift three. Left panel: feedback run. Right panel: no-feedback run. Top row plots show the baryonic ratio for plotted galaxies as a function of their total mass, while the second and bottom row plots correspond to their gas and stars mass ratio as a function of total mass. Halos with stars (crosses) and without (open circles) are shown. The dashed line corresponds to the ratio $\Omega_{b} / \Omega_{m}$. 


\section{feedback run no feedback run}
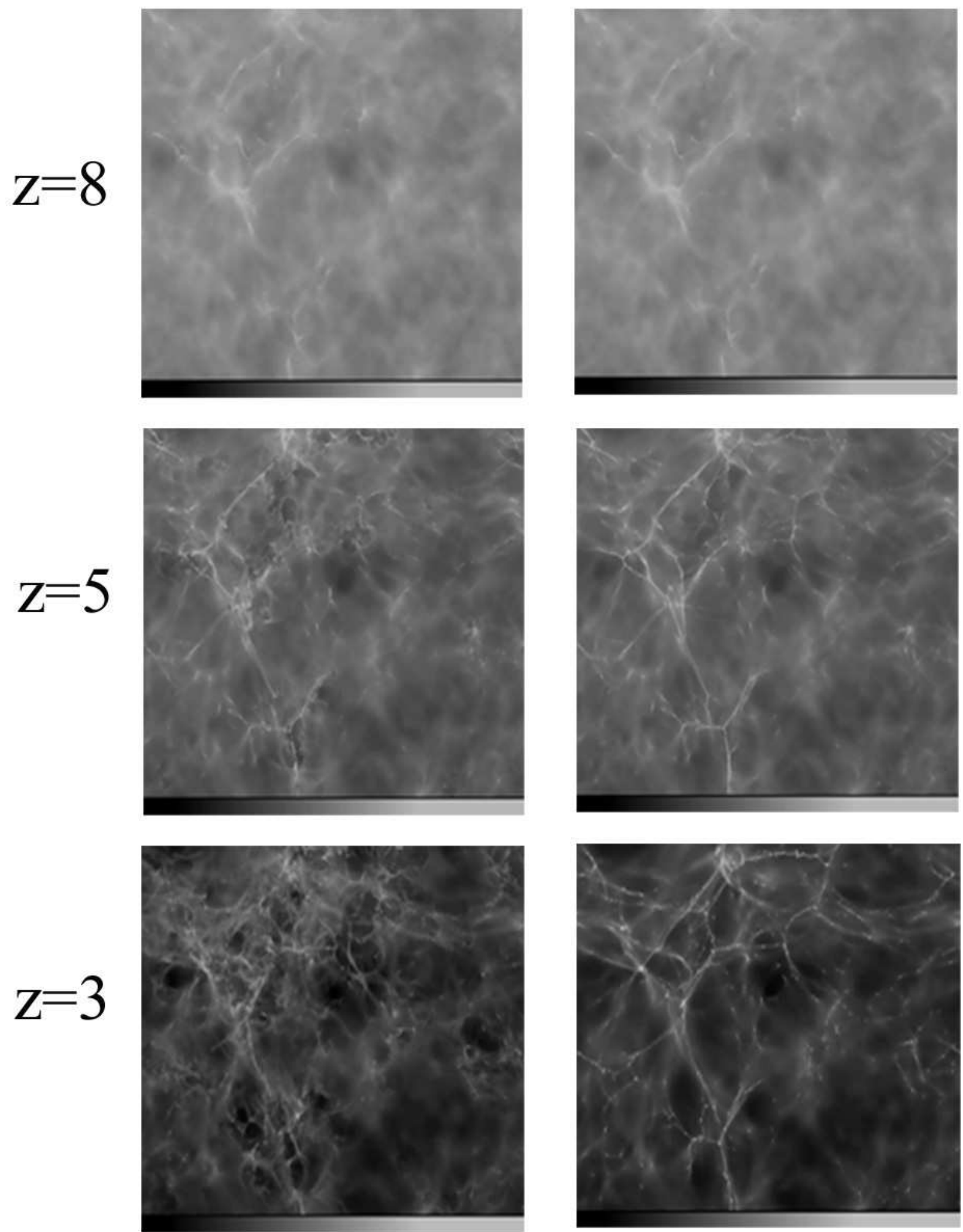

Fig. 3.- Gas density projection along x-axis. Range plotted: $11.1 \leq \log \frac{\rho_{\mathrm{gas}}}{M_{\odot} /\left(\mathrm{pr}_{\mathrm{Mpc}} \mathrm{Mp}^{2}\right.} \leq 13.6$. 


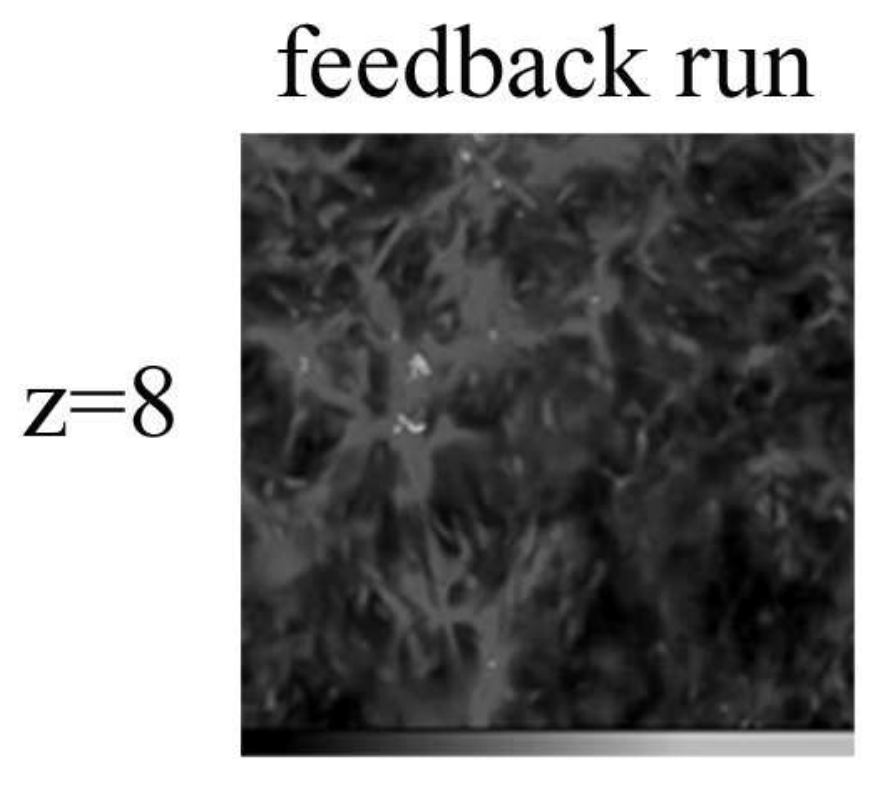

\section{no feedback run}
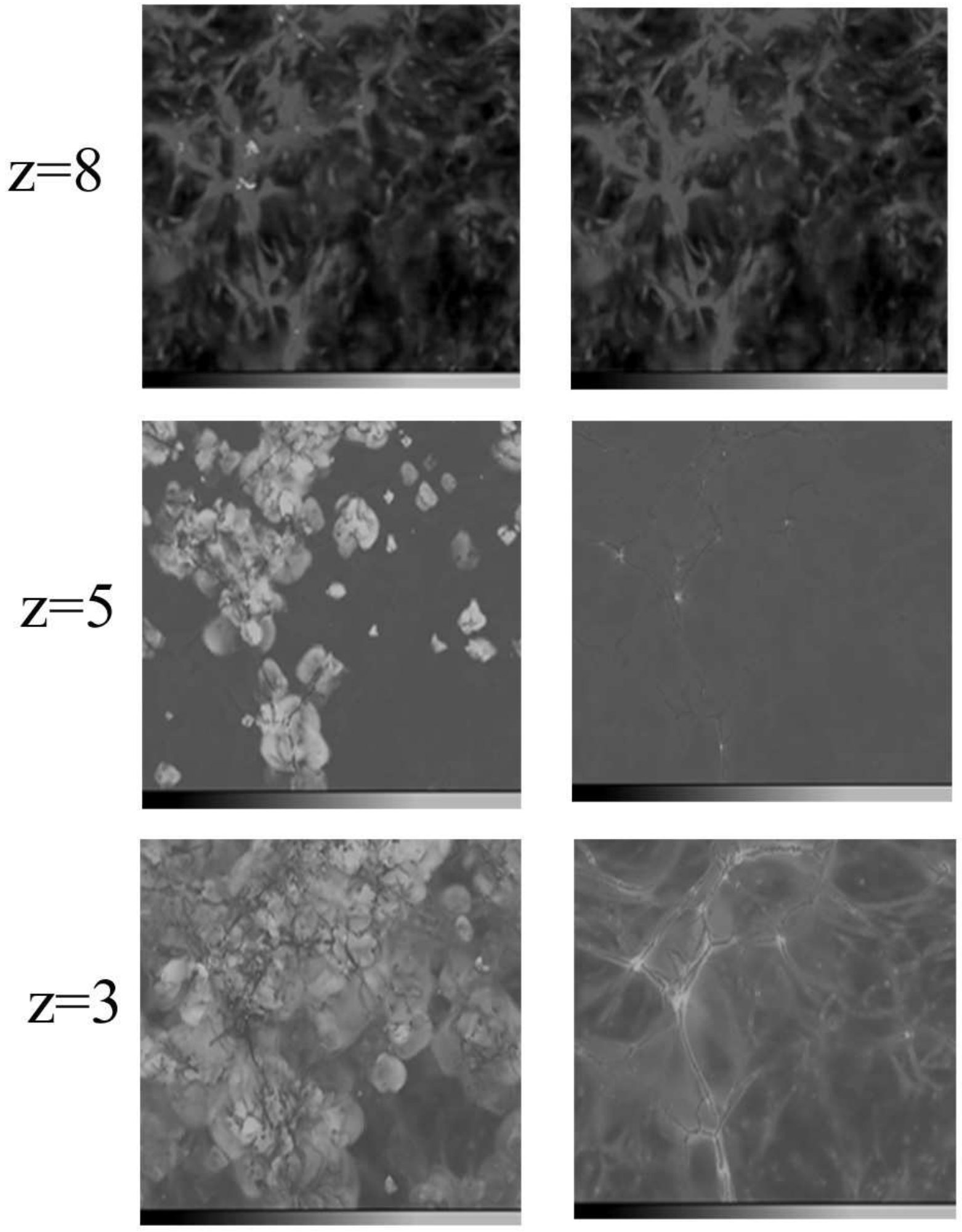

Fig. 4.- X-ray weighted temperature projection along x-axis. Range: $2.0 \leq \log \frac{T}{K} \leq 7.7$. 


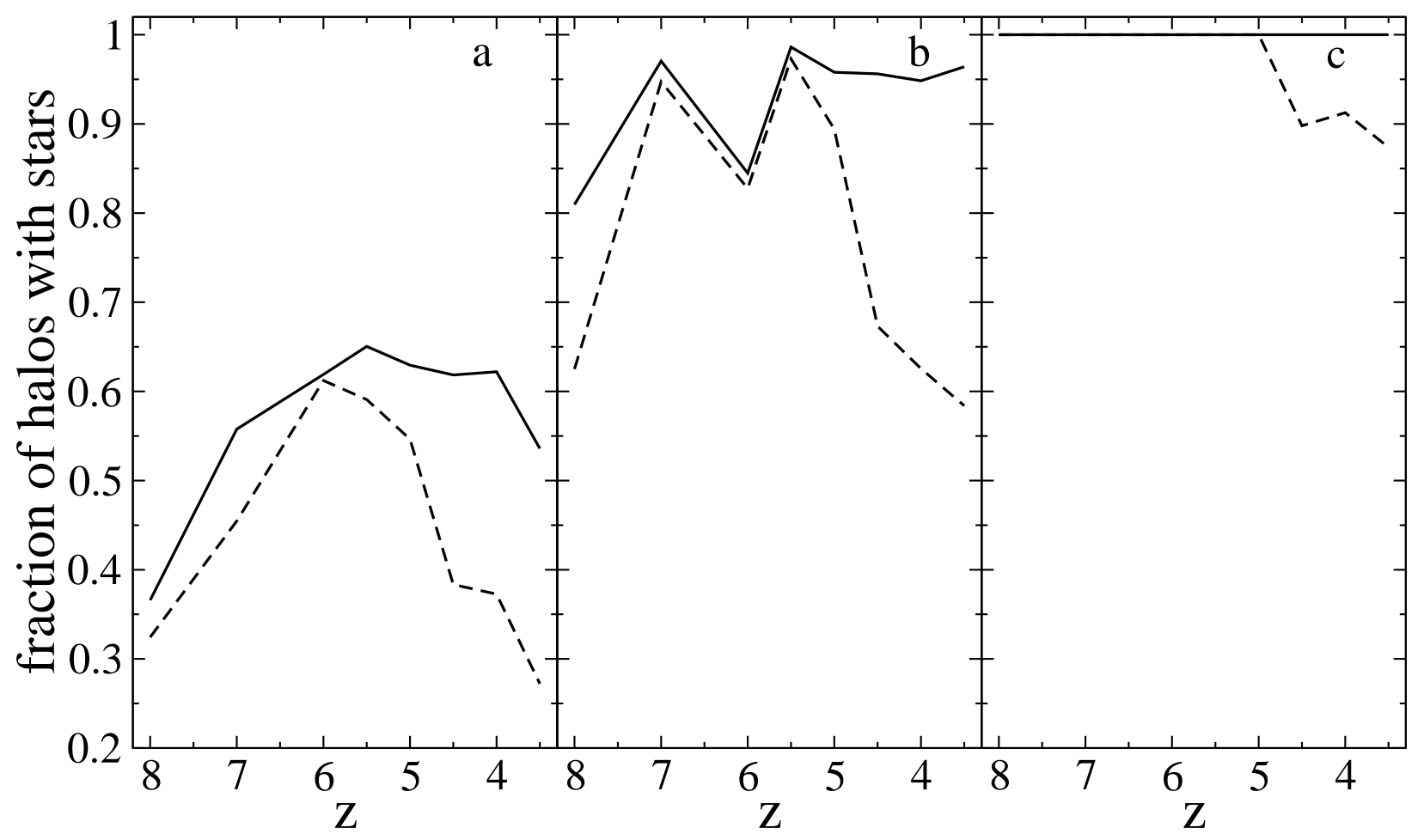

Fig. 5.- Fraction of halos hosting stars vs redshift, for 3 different mass ranges. Panel a: $10^{9} \mathrm{M}_{\odot} \leq M_{\text {total }}<3 \times 10^{9} \mathrm{M}_{\odot}$. Panel b: $3 \times 10^{9} \mathrm{M}_{\odot} \leq M_{\text {total }}<6 \times 10^{9} \mathrm{M}_{\odot}$. Panel c: $6 \times 10^{9} \mathrm{M}_{\odot} \leq M_{\text {total }}<2 \times 10^{10} \mathrm{M}_{\odot}$. Solid line curves correspond to the no-feedback run, while dashed line curves correspond to the feedback run. 


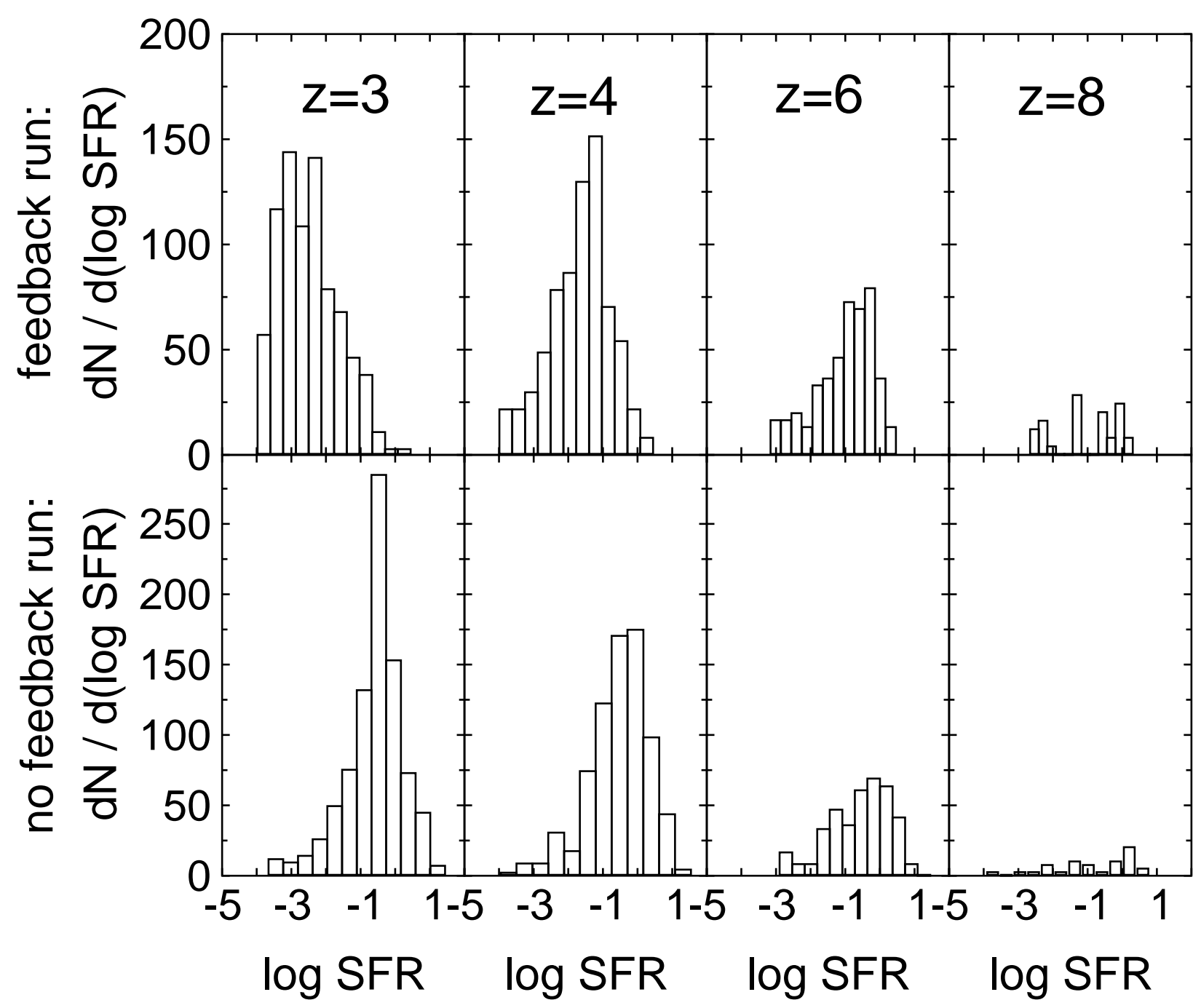

Fig. 6.- Distribution of galaxies per logarithmic star formation rate interval. Star formation rates are measured in units of $\mathrm{M}_{\odot} \mathrm{yr}^{-1}$. The top panel corresponds to the no-feedback run, while the bottom panel corresponds to the run with stellar feedback.Results for $\mathrm{z}=3,4,6$ and 8 (from left to right) are shown. 


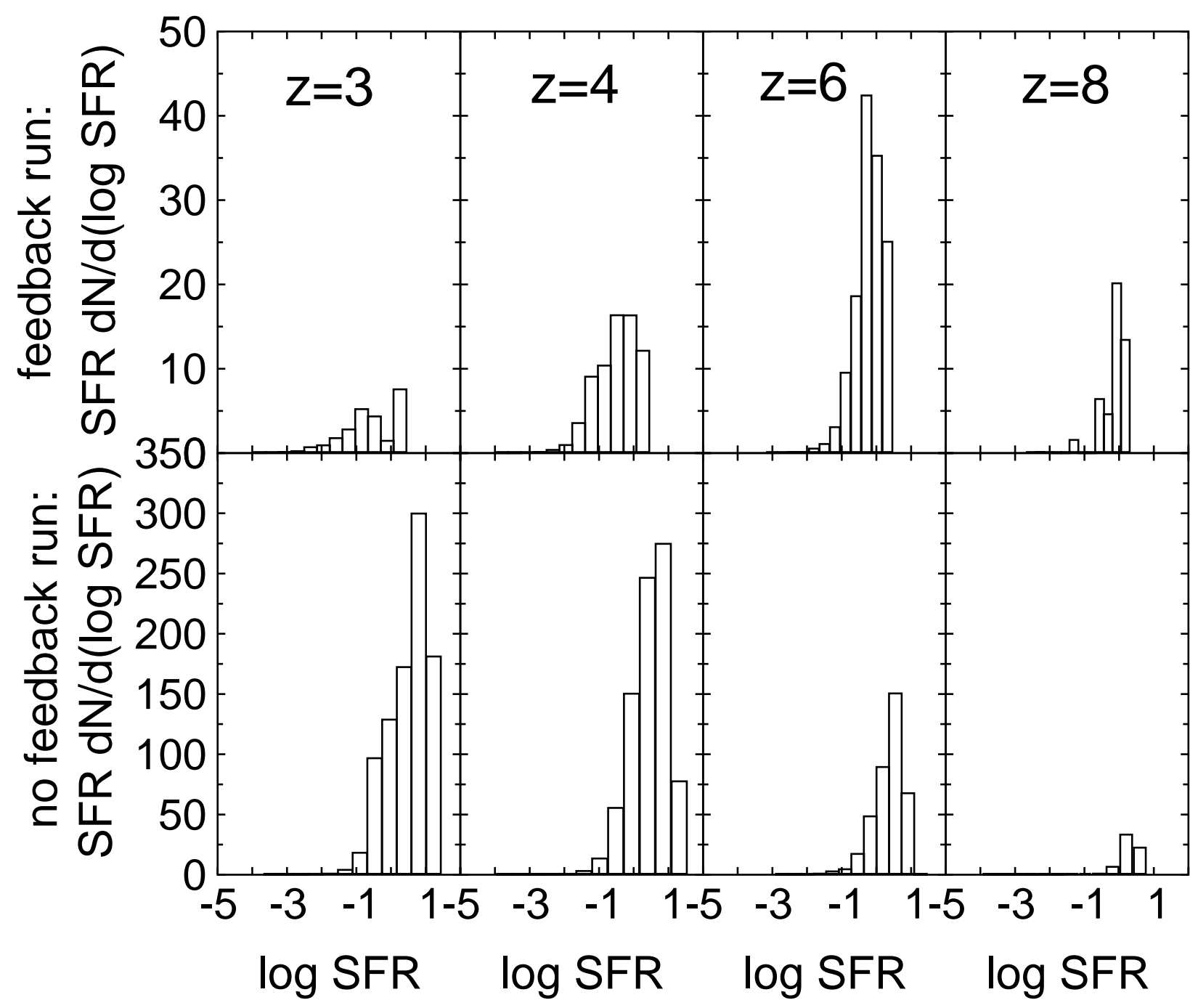

Fig. 7. $-\mathrm{SFR} \times \frac{\mathrm{dN}}{\mathrm{d} \log \mathrm{SFR}}$ as a function of $\log \mathrm{SFR}$. The area under the curve plotted is the total SFR in the simulated cube. 


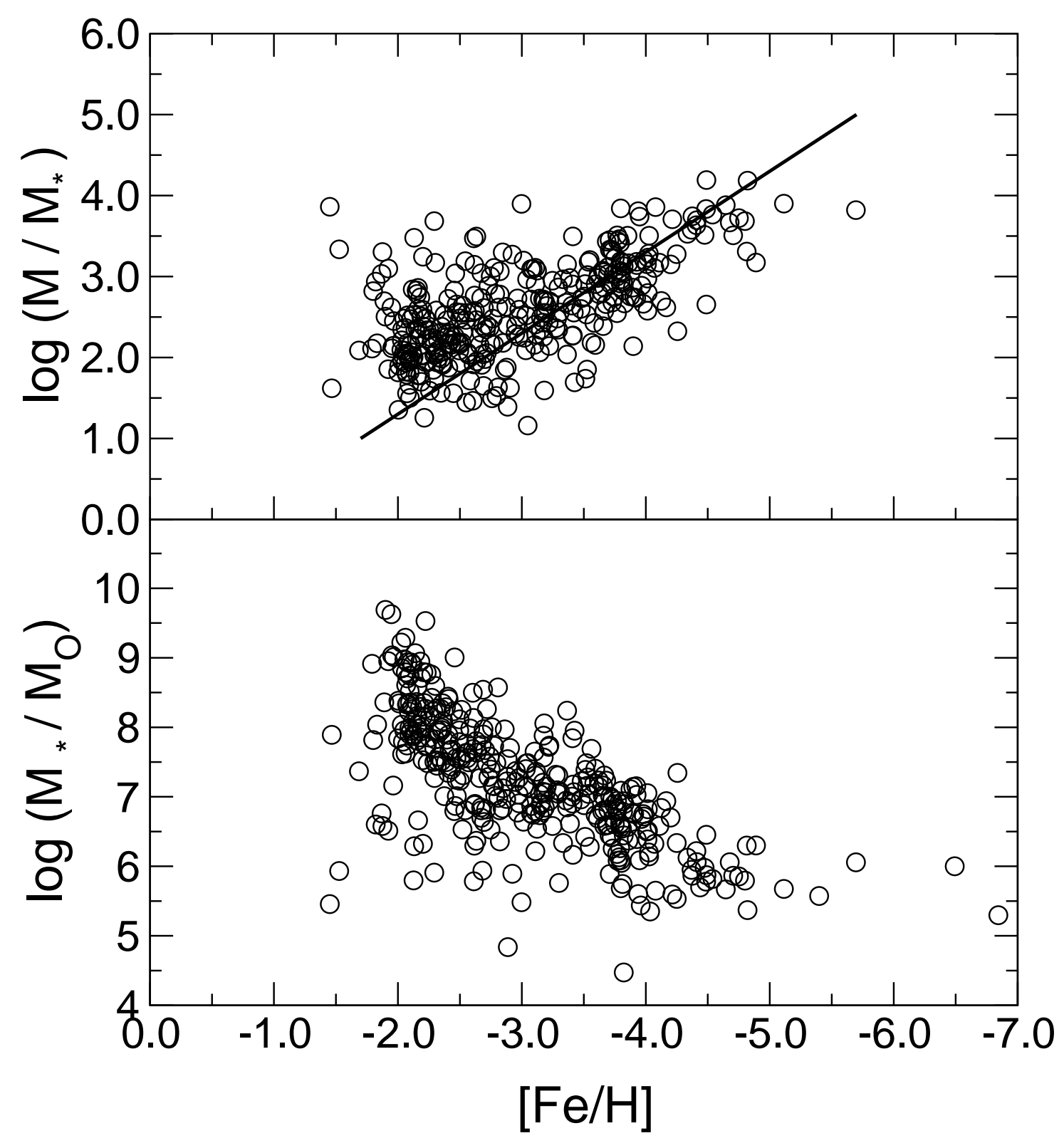

Fig. 8.- Upper panel: $\log \left(M_{\text {total }} / M_{\text {stars }}\right)$ vs mass weighted average metallicity of stars in each halo. A clear trend for decreasing metallicity with increasing $M_{\text {total }} / M_{\text {stars }}$ is found. The solid line indicates the slope of the same trend found in observations of dwarf spheroidal galaxies in the Local Group (Prada \& Burkert 2002). Lower panel: stellar masses of halos vs their metallicities. 